\title{
Parasitismo de ovos de Leptopharsa heveae Drake \& Poor por Erythmelus tingitiphagus (Soares) em plantios de seringueira com aplicação de produtos fitossanitários
}

\author{
Rodrigo Souza Santos ${ }^{1}$
}

\begin{abstract}
RESUMO
O percevejo-de-renda Leptopharsa heveae Drake \& Poor (Hemiptera: Tingidae) é uma das principais pragas da heveicultura no Brasil, podendo causar prejuízos de até 30\% na produtividade de látex. Seu controle é realizado principalmente com uso de produtos fitossanitários e uma das alternativas a seu uso seria a utilização de inimigos naturais. O parasitoide Erythmelus tingitiphagus (Soares) (Hymenoptera: Mymaridae) é um importante inimigo natural de L. heveae, por parasitar seus ovos em condições naturais. O objetivo deste estudo foi verificar a ocorrência e o parasitismo de E. tingitiphagus, em ovos de L. heveae, em talhões comerciais de cinco clones de seringueira onde se realizaram aplicações regulares de produtos fitossanitários, no município de Itiquira, MT. Semanalmente, entre agosto de 2006 a janeiro de 2007, foram coletadas aleatoriamente quatro folhas maduras em cinco árvores dos clones RRIM 600, PR 255, GT 1, PB 235 e PB 217. Na área de estudo, E. tingitiphagus ocorreu em todos os clones estudados, com pico populacional em outubro de 2006. A porcentagem de parasitismo nos diferentes clones variou de $13,8 \%$ no clone PB 235 a 30,8\% no RRIM 600 e a porcentagem média de parasitismo foi de $24,2 \%$. Os produtos fitossanitários, no método em que foram aplicados nos talhões, não atuaram negativamente no parasitismo de E. tingitiphagus.
\end{abstract}

Palavras-chave: Chalcidoidea, controle biológico, heveicultura, parasitoide de ovos.

\section{ABSTRACT \\ Parasitism of Leptopharsa heveae Drake \& Poor eggs by Erythmelus tingitiphagus (Soares) in rubber tree plantations with application of pesticides}

The rubber tree lace bug, Leptopharsa heveae Drake \& Poor (Hemiptera: Tingidae), is one of the main rubber tree crop pests in Brazil. It can cause loss of up to $30 \%$ in latex yield. The control is performed mainly with pesticides and one of the alternatives to their use is the utilization of natural enemies. The parasitoid Erythmelus tingitiphagus (Soares) (Hymenoptera: Mymaridae) has been shown to be an important natural enemy of L. heveae, parasitizing eggs of this pest in natural conditions. The aim of this study was to verify the occurrence and the parasitism of $E$. tingitiphagus on L. heveae eggs in commercial stands of five rubber tree clones which are regularly treated with pesticide, in the municipality of Itiquira, Mato Grosso State, Brazil. Weekly, between August 2006 and January 2007, four mature leaves were randomly sampled in five trees of the clones RRIM 600, PR 255, GT 1, PB 235 and PB 217. Parasitoids were found in all studied clones with populations peaking in October. The parasitism rate ranged from $13.8 \%$ in PB 235 to $30.8 \%$ in RRIM 600, with average of $24.2 \%$. The application of pesticides in the stands had no negative effect onon parasitism of E. tingitiphagus.

Key words: Chalcidoidea, biological control, rubber tree crop, egg parasitoid.

Recebido para publicação em 11/08/2011 e aprovado em 12/05/2014.

'Biólogo, Doutor. Laboratório de Entomologia, CPAFAC/EMBRAPA, Rodovia BR-364, Km 14, 69900-970, Caixa Postal 321, Rio Branco, Acre, Brasil. rodrigo.s.santos@embrapa.br 


\section{INTRODUÇÃO}

Hevea brasiliensis Müell. Arg. (Euphorbiaceae) é uma planta de origem tropical (bacia Amazônica) cultivada e utilizada de modo extrativista com a finalidade de produção de borracha natural (Campelo Júnior, 2000). A partir da introdução de sementes e/ou mudas em outros países, passou a ser cultivada em grandes monoculturas, principalmente nos países asiáticos. No Brasil, seu cultivo teve grande sucesso nos estados de São Paulo, Mato Grosso, Bahia, Espírito Santo e Paraná (Marinho, 2006). Pino et al. (2000) ressaltam que a industrialização do látex é um segmento importante da economia agrícola e industrial do Brasil.

Com o aumento da área plantada de seringueira e a adoção da monocultura em áreas extensivas a incidência de ataque de pragas se tornou mais comum. Dentre elas, destaca-se o percevejo-de-renda, Leptopharsa heveae Drake \& Poor (Hemiptera: Tingidae), cujas ninfas e adultos sugam a seiva das folhas, o que diminui sua atividade fotossintética, podendo reduzir a produção de látex em até 30\% (Tanzini \& Lara, 1998).

São relatados como principais inimigos naturais de $L$. heveae os crisopídeos (Scomparin, 1997), o fungo entomopatogênico Sporothrix insectorum (Hoog \& Evans) (Tanzini, 2002) e o mimarídeo Erythmelus tingitiphagus (Soares) (Hymenoptera: Mymaridae) (Costa et al., 2003; Santos \& Freitas, 2008), dentre outros. Este último é um parasitoide de ovos, apresentando dimensões extremamente reduzidas (0,44 e 0,51 mm de comprimento para machos e fêmeas, respectivamente) (Soares, 1941). Ocorre no Brasil, Venezuela e Argentina e, além de L. heveae, parasita ovos dos tingídeos Corythaica cyathicollis (Costa), Corythaica monacha (Stäl), Gargaphia lunulata (Mayr) e Leptodictya tabida (Herrich-Schaeffer) (Triapitsyn, 2003).

Santos \& Freitas (2008) verificaram uma porcentagem de parasitismo média de $18,8 \%$ em ovos de $L$. heveae parasitados por E. tingitiphagus em condições naturais, em clones de seringueira, cultivados em sistema policlonal e que se encontravam isentos da aplicação de produtos fitossanitários por três anos. Entretanto, produtos fitossanitários ainda são frequentemente utilizados no controle de L. heveae, o que pode provocar desequilíbrio biológico, pela eliminação de organismos benéficos presentes no complexo biológico do ecossistema, além de contribuir na seleção de populações de insetos-praga resistentes e gerar contaminação ambiental (Gallo et al., 2002).

O objetivo deste estudo foi verificar a ocorrência e a porcentagem de parasitismo de E. tingitiphagus em ovos de L. heveae, em talhões comerciais de seringueira, tratados regularmente com produtos fitossanitários e fungos entomopatogênicos, visando o controle do percevejo-derenda.

\section{MATERIAL E MÉTODOS}

O estudo foi conduzido entre agosto de 2006 a janeiro de 2007 em talhões comerciais de seringueira, cultivados com os clones RRIM 600, GT 1, PR 255, PB 235 e PB 217 , em área comercial da empresa Plantações Edouard Michelin Ltda. (17²2'23”S, 5444'23”W), no município de Itiquira, MT.

Os talhões estudados possuem área aproximada de 10 ha e foram cultivados em regime tradicional de monocultivo, com espaçamento de 2,5 m entre árvores e $8 \mathrm{~m}$ entre as linhas (Tabela 1).

Durante o período de estudo, as árvores sofreram aplicações periódicas de produtos fitossanitários, com, ou sem, a incorporação de fungos entomopatogênicos na calda. Os produtos utilizados foram: Monocrotophós ${ }^{\circledR}$, Monocrotophós ${ }^{\circledR}+S$. insectorum, Monocrotophós ${ }^{\circledR}+$ Paecilomyces sp., Metamidophós ${ }^{\circledR}$, Metamidophós ${ }^{\circledR}+$ Paecilomyces sp., Methomyl ${ }^{\circledR}+$ Paecilomyces sp.

As amostragens consistiam de coletas semanais de folhas maduras (completamente expandidas) localizadas no terço inferior da copa, que apresentavam sintomas de ataque de $L$. heveae, em árvores dos cinco clones estudados, totalizando 24 coletas no período. De acordo com Cividanes et al. (2004), adultos e ninfas de L. heveae se distribuem uniformemente nos diferentes estratos da planta, não comprometendo a amostragem vertical. Foram escolhidas, de forma aleatória, cinco árvores por clone, de onde eram coletadas quatro folhas, totalizando 20 folhas por clone/coleta. As folhas coletadas eram acondicionadas em sacos de papel identificados e levadas ao laboratório, onde os folíolos eram preparados conforme metodologia utilizada por Santos \& Freitas (2008).

A avaliação ocorreu sete dias após a montagem dos ensaios, observando o interior dos sacos plásticos e folíolos sob microscópio estereoscópico, e contabilizando o número de parasitoides emergidos. Com auxílio de instrumento pontiagudo, os ovos de L. heveae não eclodidos, foram dissecados à procura de parasitoides não emergidos. A porcentagem de parasitismo foi obtida pelo número total de indivíduos obtidos por clone (inclusive os não

Tabela 1. Características dos clones de seringueira nos talhões estudados, no período de agosto de 2006 a janeiro de 2007, em Itiquira, MT.

\begin{tabular}{lccc}
\hline Talhão & Clone & $\begin{array}{c}\text { Idade } \\
\text { das árvores } \\
\text { (anos) }\end{array}$ & $\begin{array}{c}\text { Altura aproximada } \\
\text { das árvores } \\
(\mathbf{m})\end{array}$ \\
\hline 308D & RRIM 600 & 26 & $14-16$ \\
412C & GT 1 & 21 & $11-13$ \\
407C & PR 255 & 21 & $11-13$ \\
611B & PB 235 & 24 & $14-16$ \\
408B & PB 217 & 24 & $14-16$ \\
\hline
\end{tabular}

Rev. Ceres, Viçosa, v. 61, n.3, p. 350-355, mai/jun, 2014 
emergidos), dividindo-se pelo número total de ovos de $L$. heveae por clone e multiplicando-se o valor por 100 .

Os dados foram submetidos à análise de variância (ANOVA) e suas médias comparadas pelo teste de Tukey a 5\% de probabilidade (Zar, 2010). Após a confirmação da distribuição normal dos dados, através de análise gráfica, foi realizado o teste de Levene (ZAR, 2010) para verificar a homogeneidade das variâncias.

Os parasitoides obtidos foram preservados em álcool (80\%) e identificados pelo Dr. Valmir Antonio Costa (Instituto Biológico/APTA, Campinas, SP).

\section{RESULTADOS E DISCUSSÃO}

Nos 2.400 folíolos analisados, foram encontrados 6.717 ovos de L. heveae e 1.664 exemplares de E. tingitiphagus. A porcentagem média de parasitismo foi de $24,2 \%$ e variou de 13,8\% no clone PB 235 a 30,8\% no RRIM 600 (Tabela 2).

$\mathrm{O}$ número de adultos de E. tingitiphagus variou de 173 exemplares no clone PB 235 a 486 no RRIM 600 (Tabela 2). A disponibilidade de hospedeiros (ovos) ao parasitoide, que variou entre os clones, certamente é um dos fatores que explica a diferença no número de parasitoides observados entre os clones.

Foi verificado que o pico populacional de $E$. tintigiphagus foi predominantemente em outubro de 2006, com exceção do clone GT 1, cujo pico ocorreu em novembro de 2006 (Figura 1). Este resultado concorda parcialmente com os obtidos por Santos \& Freitas (2008), que relataram o pico populacional de E. tingitiphagus em novembro, em área não tratada. No entanto, pelos resultados obtidos nestas pesquisas realizadas na mesma área, constata-se que os picos populacionais do parasitoide ocorrem entre os meses de outubro a novembro.

Observou-se que o número médio mensal de ovos de L. heveae por folíolo foi pequeno no início das amostragens (agosto de 2006), atingindo o pico populacional máximo em outubro de 2006, e diminuindo sensivelmente entre novembro de 2006 a janeiro de 2007 (Figura 2). A predominância de folhas novas no mês de agosto de 2006, provavelmente interferiu negativamente na

Tabela 2. Número de Erythmelus tingitiphagus, ovos de Leptopharsa heveae e porcentagem de parasitismo em cinco clones de seringueira, no período de agosto de 2006 a janeiro de 2007 , em Itiquira, MT.

\begin{tabular}{lccc}
\hline Clones & $\begin{array}{c}\text { Ovos } \\
(\mathbf{n})\end{array}$ & $\begin{array}{c}\text { Parasitoides } \\
(\mathbf{n})\end{array}$ & $\begin{array}{c}\text { Parasitismo } \\
(\%)\end{array}$ \\
\hline RRIM 600 & 1.576 & 486 & 30,8 \\
PR 255 & 1.571 & 430 & 27,4 \\
PB 217 & 1.247 & 336 & 26,9 \\
GT 1 & 1.071 & 239 & 22,3 \\
PB 235 & 1.252 & 173 & 13,8 \\
\hline Total & 6.717 & 1.664 & $\bar{x}=24,2$ \\
\hline
\end{tabular}

oviposição de $L$. heveae, o que concorda com Santos (2007), que observou maior quantidade de oviposições de $L$. heveae em folhas maduras, em relação às novas e de fenologia intermediária. Possivelmente as características físico-químicas relacionadas à fenologia das folhas, interfiram positiva e/ou negativamente na biologia de $L$. heveae, e confiram diferenças na resistência dos clones ao ataque de $L$. heveae.

O número médio mensal de parasitoides adultos (Figura 1) acompanhou a variação do número de ovos de $L$. heveae (Figura 2) e apresentou pico populacional em outubro de 2006, quando a disponibilidade de hospedeiros (ovos) foi maior. A ação sinérgica dos fatores: (1) pulverização dos produtos fitossanitários nos talhões, (2) aumento populacional de E. tingitiphagus e (3) fatores meteorológicos na região, são as possíveis causas da diminuição da população de $L$. heveae nos meses de novembro de 2006 a janeiro de 2007. Com relação à população do parasitoide, a aplicação dos produtos fitossanitários causando mortalidade nos adultose o declínio populacional do hospedeiro são fatores que possivelmente contribuíram para seu declínio populacional.

As médias máximas mensais de ovos por folíolo nos clones foram de 8,7 (RRIM 600); 5,7 (PR 255); 4,6 (PB 217); 4,1 (GT 1) e 3,6 (PB 235) (Figura 2). Estes valores foram inferiores aos verificados por Santos \& Freitas (2008), em talhões dos mesmos clones que não sofriam pulverizações. Como o esperado, as aplicações dos produtos fitossanitários influenciaram negativamente na população do percevejo-de-renda, causando a mortalidade dos adultos, o que resultou em menor número de oviposições. Estas aplicações proporcionaram uma maior redução na população da praga ( $\approx 20 \%$ ) em relação à do parasitoide, quando comparamos aos resultados obtidos por Santos \& Freitas (2008) em área não pulverizada.

O clone GT 1 apresentou o menor número médio de ovos por folíolo, enquanto RRIM 600 e PR 255 apresentaram os maiores. As médias máximas mensais de $E$. tingitiphagus por folíolo nos clones foram de 3,3 (RRIM 600); 2,2 (PR 255); 1,7 (PB 127); 1,2 (GT 1) e 0,8 (PB 235) (Figura 1).

A aplicação regular de produtos fitossanitários nos talhões proporcionou redução no número de oviposições ( $\approx 36 \%$ ) de L. heveae e no número de parasitoides obtidos $(\approx 16 \%$ ), quando comparada aos números observados por Santos \& Freitas (2008) em área não pulverizada. No entanto, em relação à porcentagem média de parasitismo, os talhões que sofreram aplicações de produtos fitossanitários apresentaram maior porcentagem de parasitismo $(24,2 \%)$ em relação ao verificado por Santos \& Freitas (2008) para os mesmos clones, em área não pulverizada $(18,8 \%)$. Tal constatação permite a propositura de algumas hipóteses: (1) os produtos aplicados na área são se- 
letivos a E. tingitiphagus; (2) o método de aplicação dos produtos (aplicação apenas no foco da infestação e não na área total do talhão) permite a manutenção da população do parasitoide na área; (3) os produtos aplicados não eliminam as posturas endofíticas de $L$. heveae, cujos ovos parasitados se mantém viáveis e proporcionam uma contínua re-colonização da área; (4) os produtos utilizados tem um período residual curto nas condições edafoclimáticas da região e (5) a eficiência de parasitismo por fêmeas de E. tingitiphagus é maior em folíolos com menor quantidade de ovos de L. heveae.

Apesar da necessidade da realização de outras pesquisas, a fim de testar cada uma destas hipóteses, algumas são mais plausíveis para explicar o alto índice de parasitismo de E. tingitiphagus, mesmo em talhões que sofrem pulverizações periódicas. O método de aplicação no foco do ataque, e não na área total do talhão, bem como o fato de os ovos endofíticos de L. heveae não sofrerem influência das pulverizações, possivelmente sejam os fatores determinantes para garantir a manutenção da população do parasitoide na área, o que explicaria as altas taxas de parasitismo observadas. Os produtos fitossanitários aplicados não se demonstraram seletivos a outras espécies de predadores e parasitoides (Gusmão et al., 2000; Bacci et al,. 2001; Perioto et al., 2002) e, provavelmente, não os seriam a $E$. tingitiphagus.

O clone PB 235 não foi o menos ovipositado por $L$. heveae, entretanto foi o que apresentou o menor número de exemplares de E. tingitiphagus e a menor porcentagem de parasitismo, enquanto que RRIM 600 apresentou os maiores índices (Tabela 2). Estes resultados concordam com os obtidos por Santos \& Freitas (2008), nos quais as menores e maiores porcentagens de parasitismo foram observados para os clones PB 235 e RRIM 600 respectivamente, em talhão sem aplicação de produtos fitossanitários. Neste sentido, sugere-se que as características intrínsecas de resistência química e/ou física entre os clones influenciem mais fortemente na oviposição de $L$. heveae e ocorrência de E. tingitiphagus, em detrimento à influência das pulverizações, nas condições em que foram realizadas. A porcentagem de ocorrência de E. tingitiphagus nos clones foi de 10,4 (PB 235); 14,4 (GT 1); 20,2 (PB 217); 25,8 (PR 255) e $29,2 \%$ (RRIM 600). Estes resultados são similares aos obtidos por Santos \& Freitas (2008) em área não pulverizada.

Substâncias químicas produzidas pelas plantas, em resposta às injúrias causadas pelas pragas, podem atuar como sinalizadores químicos para parasitoides (Thaler, 1999). A concentração destes semioquímicos pode dife-

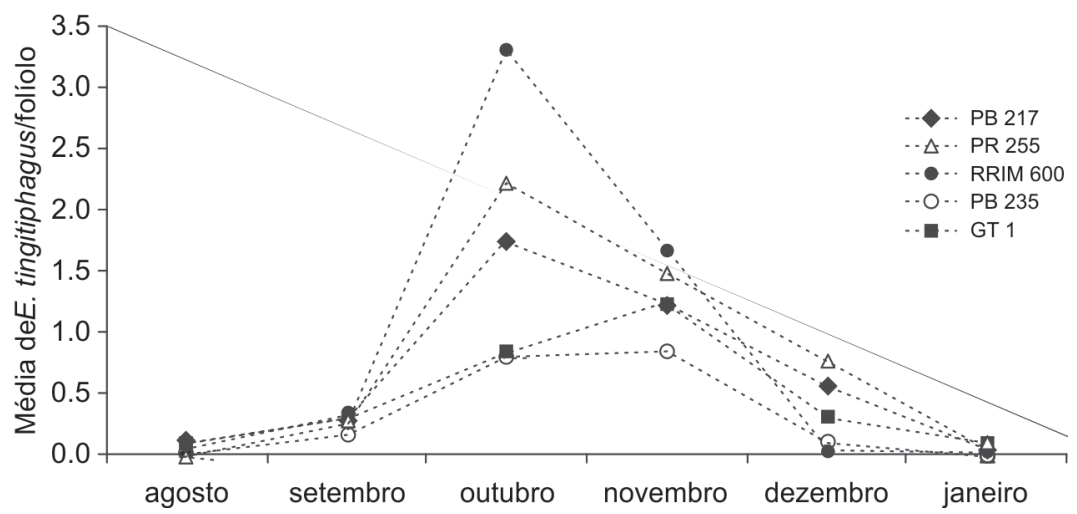

Figura 1. Número médio mensal de Erythmelus tingitiphagus por folíolo, em cinco clones de seringueira, no período de agosto de 2006 a janeiro de 2007, em Itiquira, MT.

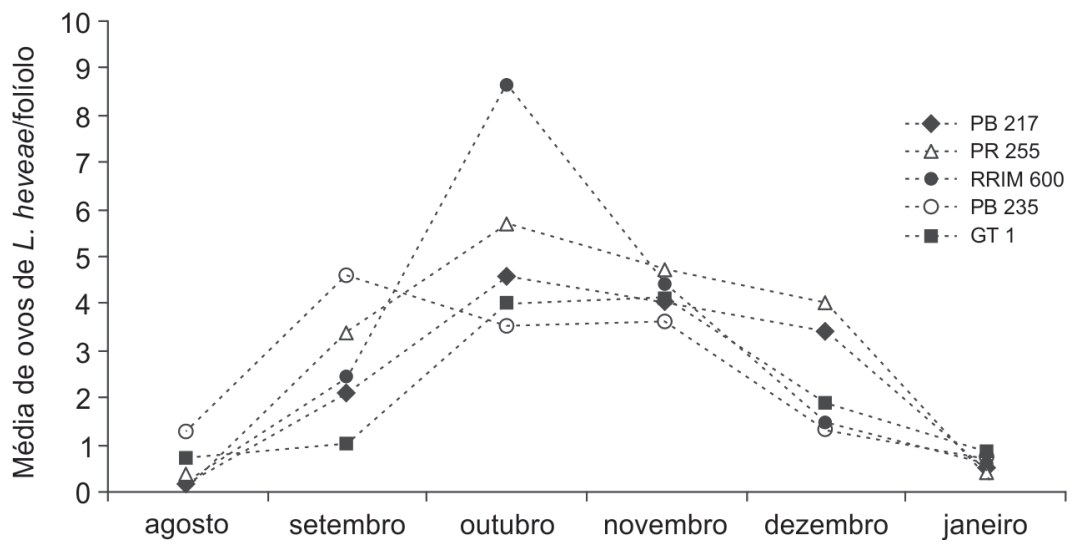

Figura 2. Número médio mensal de ovos de Leptopharsa heveae por folíolo, em cinco clones de seringueira, no período de agosto de 2006 a janeiro de 2007, em Itiquira, MT. 
rir entre os clones estudados, o que poderia explicar a menor atratividade de parasitoides pelo clone PB 235. Além disso, a característica das folhas (principalmente a espessura da cutícula) e a arquitetura das árvores dos clones estudados podem proporcionar condições microclimáticas relativamente favoráveis ao inimigo natural, podendo influenciar na ocorrência do mesmo (Ganzhorn, 1995; Price et al., 1995). A presença de nectários extraflorais em alguns clones de seringueira tais como RRIM 600 e PR 255 (Gonçalves, 2002) também podem influenciar na maior ocorrência de parasitoides nestes clones, já que proporcionam uma fonte alimentar suplementar para os adultos.

Apesar de haverem diferenças em relação ao número de oviposições de L. heveae, no número de parasitoides e na porcentagem de parasitismo entre os clones estudados, não foi constatada diferença estatística entre os mesmos (Tabela 3). Este resultado demonstra que $E$. tingitiphagus atua de forma homogênea, independente do clone de seringueira, atuando como um importante inimigo natural de L. heveae, mesmo em talhões que sofrem pulverizações periódicas.

Os resultados obtidos indicam que este parasitoide tem potencial para ser utilizado em um sistema de manejo integrado de $L$. heveae em seringais de cultivo, concomitante ao emprego de produtos fitossanitários. Entretanto, serão necessários estudos de seletividade dos produtos utilizados, bem como estudos para dar suporte à criação massal de E. tingitiphagus em laboratório e estudos que visem as estratégias de liberação em campo.

Tabela 3. Médias de Erythmelus tingitiphagus, ovos de Leptopharsa heveae e porcentagem de parasitismo, em cinco clones de seringueira, no período de agosto de 2006 a janeiro de 2007, em Itiquira, MT.

\begin{tabular}{lccc}
\hline Clones & E. tingitiphagus $(\bar{x})$ & L. heveae $(\bar{x})$ & \% Parasitismo $(\bar{x})$ \\
\hline RRIM 600 & $20,25^{\mathrm{a}}$ & $65,66^{\mathrm{a}}$ & $0,18^{\mathrm{a}}$ \\
PR 255 & $17,91^{\mathrm{a}}$ & $65,45^{\mathrm{a}}$ & $0,16^{\mathrm{a}}$ \\
PB 217 & $14,00^{\mathrm{a}}$ & $51,95^{\mathrm{a}}$ & $0,17^{\mathrm{a}}$ \\
GT 1 & $9,95^{\mathrm{a}}$ & $44,62^{\mathrm{a}}$ & $0,20^{\mathrm{a}}$ \\
PB 235 & $7,20^{\mathrm{a}}$ & $52,16^{\mathrm{a}}$ & $0,11^{\mathrm{a}}$ \\
\hline
\end{tabular}

As médias nas colunas com a mesma letra não diferem entre si pelo teste de Tukey (5\%).

\section{CONCLUSÕES}

As menores e maiores porcentagens de parasitismo e ocorrência de E. tingitiphagus ocorreram nos clone PB 235 e RRIM 600, respectivamente.

Os picos populacionais do parasitoide e do percevejo-de-renda foram registrados em outubro de 2006 na área estudada.

Os produtos fitossanitários, na forma que foram aplicados nos talhões, não atuaram negativamente no parasitismo de E. tingitiphagus.

\section{AGRADECIMENTOS}

À mestranda Luciana Maira de Sales Pereira (Universidade Federal do Acre - UFAC) pela revisão do texto e Abstract. Ao MSc. Joaquim Manoel da Silva (Universidade do Estado de Mato Grosso - UNEMAT, Campus de Nova Xavantina, MT) pelo auxílio nas análises estatísticas e pelas sugestões ao manuscrito.

\section{REFERÊNCIAS}

Bacci L, Picanço MC, Gusmão MR, Crespo ALB \& Pereira EJG (2001) Seletividade de inseticidas a Brevicoryne brassicae (L.) (Hemiptera: Aphididae) e ao predador Doru luteipes (Scudder) (Dermaptera: Forficulidae). Neotropical Entomology, 30:707-713.

Campelo Júnior JH (2000) Estimativa da transpiração em seringueira. Revista de Agrometeorologia, 8:35-42.
Cividanes FJ, Fonseca FS \& Santos TM (2004) Distribuição de Leptopharsa heveae em seringal do Estado de São Paulo. Pesquisa Agropecuária Brasileira, 39:1053-1056.

Costa VA, Pereira C de F \& Batista Filho A (2003) Observações preliminares sobre o parasitismo de ovos de Leptopharsa heveae (Hemiptera: Tingidae) em seringueira em Pindorama, SP. Arquivos do Instituto Biológico, 70:205-206.

Gallo D, Nakano O, Silveira Neto S, Baptista GC, Berti Filho E, Parra JRP, Zucchi RA, Alves SB, Vendramim JD, Marchini LC, Lopes JRS \& Omoto C (2002) Entomologia agrícola. Piracicaba, Fealq. 920p.

Ganzhorn JU (1995) Low-level forest disturbance effects on primary production, leaf chemistry, and lemur populations. Ecology, 76:2084-2096.

Gonçalves P de S (2002) Sub-produtos complementares da renda de um seringal. Disponível em: 〈http://www.apabor.or.br >. Acessado em: 12 de maio de 2014.

Gusmão MR, Picanço MC, Gonring AHR \& Moura MF (2000) Seletividade fisiológica de inseticidas a Vespidae predadores do bicho-mineiro-do-cafeeiro. Pesquisa Agropecuária Brasileira, 35:681-686.

Marinho JTS (2006) Seringueira: opções de cultivo e geração de renda na Amazônia. Disponível em: <http:// www.cpafac.embrapa.br/chefias/cna/artigos/seringa>. Acessado em: 12 de maio de 2014.

Perioto NW, Lara RIR, Santos JCC, Selegatto A \& Luciano ES (2002) Seletividade de thiamethoxam sobre a entomofauna de himenópteros parasitóides (Insecta, Hymenoptera) na cultura do feijão (Phaseolus vulgaris L.) em Ribeirão Preto, SP. Arquivos do Instituto Biológico, 69:29-32. 
Pino FA, Santos VLF dos, Martin NB \& Cortez JV (2000) Perfil da heveicultura no Estado de São Paulo. Informações Econômicas, 30:07-40.

Price PW, Andrade I, Pires C, Sujii E \& Vieira EM (1995) Gradient analysis using plant modular structure: pattern in plant architecture and insect herbivore utilization. Environmental Entomology, 24:497-505.

Santos RS (2007) Parasitismo de ovos de Leptopharsa heveae Drake \& Poor, 1935 (Hemiptera: Tingidae) em seringueira (Hevea brasiliensis Müell. Arg.) no Estado do Mato Grosso. Tese de Doutorado. Faculdade de Ciências Agrárias e Veterinárias, Jaboticabal. 114p.

Santos RS \& Freitas S de (2008) Parasitismo de Erythmelus tingitiphagus (Soares) (Hymenoptera: Mymaridae) em ovos de Leptopharsa heveae Drake \& Poor (Hemiptera: Tingidae), em plantios de seringueira (Hevea brasiliensis Müell. Arg.). Neotropical Entomology 37:571-576.

Scomparin CHJ (1997) Estudo dos crisopídeos (Neuroptera: Chrysopidae) em seringueira (Müell. Arg.), aspectos biológicos e potencial no controle biológico de Leptopharsa heveae Drake \& Poor (Hemiptera: Tingidae). Dissertação de Mestrado. Faculdade de Ciências Agrárias e Veterinárias, Jaboticabal. 173p.
Soares OM (1941) Notas sobre parasitos do tomateiro, contendo a diagnose de "Anaphes tingitiphagus", n. sp. (Himenoptera: Chalcidoidea). Boletim da Escola Nacional de Agronomia, 2:259267

Tanzini MR \& Lara FM (1998) Biologia do percevejo-de-rendada-seringueira Leptopharsa heveae Drake \& Poor (Heteroptera: Tingidae). Ecossistema, 23:65-67.

Tanzini MR (2002) Controle do percevejo-de-renda-da-seringueira (Leptopharsa heveae) com fungos entomopatogênicos. Tese de Doutorado. Escola Superior de Agricultura "Luiz de Queiroz", Piracicaba.140p.

Thaler JS (1999) Jasmonic acid mediated interactions between plants, herbivores, parasitoids and pathogens: a review of field experiments in tomato. In: Agrawal AA, Tuzun S \& Bent E (Eds.) Induced plant defenses against pathogens and herbivores: biochemistry, ecology and agriculture. Saint Paul, American Phytopathological Society Press. p.319-334.

Triapitsyn SV (2003) Review of the Mymaridae (Hymenoptera: Chalcidoidea) of Primorskii Krai: genus Erythmelus Enock, with taxonomic notes on some extralimital species. Far Eastern Entomologist, 126:01-44.

Zar JH (2010) Biostatistical analysis. 5 ${ }^{\mathrm{a}}$ ed. New Jersey, Prentice Hall. 944p. 